\title{
Hydrodynamic Singularities
}

\author{
Jens Eggers \\ Universität Gesamthochschule Essen, Fachbereich Physik, \\ 45117 Essen, Germany
}

\section{Introduction}

The equations of hydrodynamics are nonlinear partial differential equations, so they include the possibility of forming singularities in finite time. This means that hydrodynamic fields become infinite or at least non-smooth at points, lines, or even fractal objects. This mathematical possibility is the price one has to pay for the enormous conceptual simplification a continuum theory brings for the description of a many-particle system. Near singularities, the microscopic structure re-emerges, as the flow changes over arbitrarily small distances. Eventually, the singularity is cut off by some microscopic length scale such as the distance between molecules.

The most fundamental question is whether the microscopic structure becomes relevant for features of the flow much larger than the microscopic ones. If this is the case, the continuum description is no longer self-consistent, but has to be supplemented by microscopic information. There are some well-known cases where singularities are artifacts of neglecting diffusive effects like viscosity in the hydrodynamic equations, and there is no more smoothing on small scales. Examples are the singularities widely believed to be produced by the three-dimensional Euler equation (Majda (1991)) or those of Hele-Shaw flows at zero surface tension (Dai, Kadanoff, and Zhou (1991)). There does not seem to be a direct correspondence between the intricate structure of spatial singularities produced by these equations and real flows. For a realistic description of experiments, some tiny amount of viscosity or surface tension needs to be added.

The reason hydrodynamic singularities have nevertheless attracted considerable attention in recent years is the observation that certain singularities have direct physical significance and are not a consequence of inadequacies of the equations. In particular, free-surface flows exhibit a rich variety of experimentally observable singularities, which are responsible for phenomena like the breakup of jets, coalescence of drops, and bubble entrainment.

\section{Physical singularities}

We attempt to divide these physical singularities into two categories. 


\subsection{Dynamical singularities}

These are singularities which are confined to a point in time. Usually, they are associated with topological transitions like the breakup of a piece of fluid into two pieces or the joining of two pieces into one. For example, the breakup of a viscous jet of fluid is driven by surface tension, which tries to reduce the surface area by diminishing the radius of the jet (Eggers (1997a)). Inertial forces constrain the motion to become more and more localized, since smaller and smaller amounts of fluid have to move. This causes the jet to break at a point in finite time. Only the smoothing effect of viscosity prevents infinite gradients from occurring before the local radius goes to zero.

As the local radius of a fluid thread becomes smaller and smaller during pinching, it inevitably reaches a microscopic scale $\ell_{\text {micro }}$ where the equations cease to be valid. For thread diameters between 10 and $100 \mathrm{~nm}$, short-ranged van der Waals forces come into play, and for even smaller diameters the concept of a sharp interface will certainly loose its meaning. Moreover, a stability analysis (Brenner, Shi, and Nagel (1994)) shows that the pinching thread is very sensitive to thermal fluctuations. This makes even threads of micron size unstable, and leads to a new structure of nested singularities, driven by microscopic fluctuations. After the thread has dissolved, new surfaces form on either side, whose rapid retraction is again governed by the Navier-Stokes equation, but with a new topology. It would appear as if the continuation to the new Navier-Stokes problem should necessarily include the microscopic length $\ell_{\text {micro }}$ at which the thread broke. This is however not the case (Eggers (1997a)), as long as one is looking at scales much larger than $\ell_{\text {micro }}$. Namely, the final stages occur on very small spatial and temporal scales, and do not affect the flow at a finite distance away from breakup. Thus the outer part of the solution can be used as a boundary condition for the new problem after breakup. A closer analysis reveals that this is sufficient to determine the new solution completely. This means that the dynamics very quickly "forget" the microscopic details of breaking, thus making a consistent hydrodynamic theory of the topological transition possible.

\section{$2.2 \quad$ Persistent singularities}

The other important category of singularities are those which exist for a period of time, being either stationary, or moving about in space like the classical example of a shock wave. At finite viscosity, a shock wave is not a true singularity, but maintains a finite width determined by the ratio of the viscosity and the shock strength. However, the width $\delta$ of the shock wave is typically of the same order as the mean free path of the gas it is moving in. What is important is thus the fact that the solution remains consistent as $\delta$ goes to zero. Indeed, the dissipation inside the shock remains finite in this limit, so on scales much larger than $\delta$ the flow field is the same as if $\delta$ were zero.

In the realm of free-surface flows, a beautiful example of a stationary singularity has been discovered recently on the surface of the viscous flow between two 
counter-rotating cylinders (Joseph et al. (1991)). As seen in Fig.1, two counterrotating cylinders are submerged in a container filled with a very viscous fluid. The relative strength of viscous forces and surface tension is measured by the capillary number

$$
C a=\frac{\eta \Omega r_{c}}{\gamma},
$$

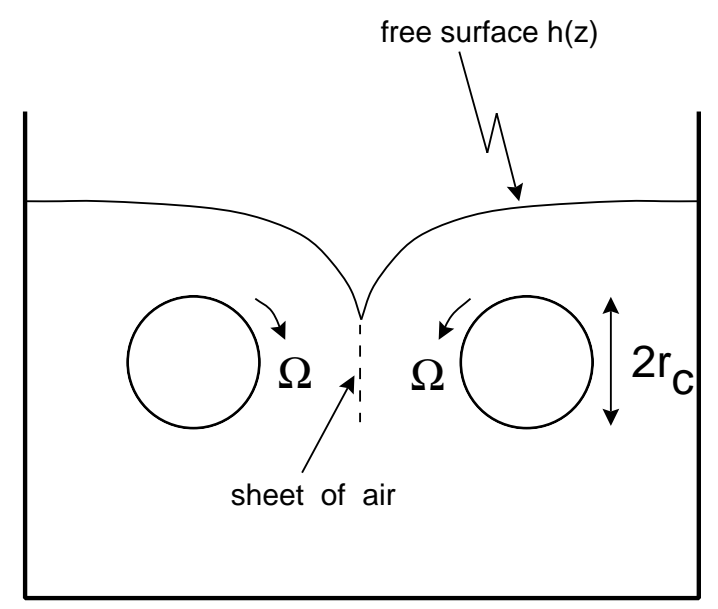

Fig. 1. Sketch of the two-roller apparatus. At a critical capillary number, a pointed cusp appears. At a second, much higher capillary number, a sheet emanates from the cusp.

where $\gamma$ is the coefficient of surface tension, $\eta$ the viscosity, and $\Omega$ and $r_{c}$ are the cylinders' rotation speed and radius, respectively. At a critical capillary number, the surface appears to form a cusp, as indicated in Fig.1. Since the flow remains two-dimensional, this corresponds to a line of singularities of the surface. Assuming that it is a true cusp, the original authors analyzed the flow using a solution due to Richardson (1968). This local solution leads to a logarithmic divergence of the dissipation, and thus cannot be consistent with continuum theory or a finite driving power. It was therefore suggested that the divergence is regularized by some microscopic scale. Since the singularity is very weak, the dissipation at the tip would still be small for realistic values of the microscopic length. However, this explanation for eliminating the logarithmic divergence would mean that there is a dependence of the macroscopic flow on a microscopic parameter.

Faced with this possibility, the problem was reanalyzed by Jeong and Moffatt (1992), who solved the Stokes equation exactly, making the simplifying assumption that the two rollers can be represented by a single dipole. The remarkable 
result of their calculation is that the radius of curvature $R$ at the tip is in fact finite, but exponentially small in the capillary number:

$$
R=R_{0} \exp [-32 \pi C a] .
$$

For realistic values of the capillary number, this gives radii of curvature far below any physical scale, but is still able to regularize the logarithmic singularity. Thus one finds a finite value of the energy dissipation, making the macroscopic flow independent of the microscopic parameters of the fluid.

For practical purposes, the theoretical value of $R$ is far too small to be realistic. Rather, it most likely is the gas above the fluid which will set the value of $R$, and this physical effect has been neglected so far. Because of the no-slip boundary condition, gas will be forced into the narrow channel formed by the cusp. A simple calculation based on lubrication theory shows that for $R=0$ this will lead to a diverging pressure at the tip of the cusp. Thus the gas will force the channel to widen to a finite radius, at which the gas pressure is comparable to the pressure inside the fluid.

It is worth noting that the independence from microscopic parameters is by no means self-evident. A famous counterexample is that of a moving contact line, which occurs for example when a circular drop is allowed to spread on the surface of a table (de Gennes (1985), Brenner and Bertozzi (1993)). Using kinematic arguments alone, one shows that there will be a logarithmic singularity of the energy dissipation at the moving contact line. There is a vast literature on this problem, dealing either with possible mechanisms for a microscopic cutoff, or with a consistent mathematical description of the resulting macroscopic dynamics. The important point to note is that continuum mechanics alone cannot resolve the problem in a self-consistent fashion. It would predict that the spreading of the drop is stalled, contradicting observation.

\section{Scaling}

The central assumption behind the description of singularities is that of locality. Their spatial and temporal scale becomes arbitrarily small, so that the dynamics should be removed from the large-scale features of the flow. However, consistency between the singular and the large scale dynamics has to be assured by matching conditions between the inner and the outer problems.

A second, closely related assumption is that of self-similarity of the singular flow, which seems a natural concept for a class of problems which lack a typical length-scale. In the case of time-dependent singularities it means that the interface shapes at different times can be mapped on one another by an appropriate rescaling of the axes. For example, the surface profile of the pinch singularity when a fluid thread breaks is (Eggers (1997a))

$$
h(z, t)=\frac{\gamma}{\eta}\left|t_{0}-t\right| \phi\left(\frac{\rho^{1 / 2}\left(z-z_{0}\right)}{\left(\eta\left|t_{0}-t\right|\right)^{1 / 2}}\right),
$$


where $t_{0}$ and $z_{0}$ are the temporal and spatial position where the fluid breaks. Remarkably, the scaling function $\phi$ is universal, independent of the type of fluid or of initial conditions. For the free surface cusp of Fig.1, the shape of the interface has the scaling form

$$
h(z)=h_{0}+R^{1 / 2} f\left(\frac{z}{R^{3 / 4}}\right),
$$

where $\left(0, h_{0}\right)$ is the position of the cusps' tip.

Naturally, it is of particular interest to compute the scaling exponents. No general understanding of what selects a particular set of exponents exists. Usually, local solutions like (3) or (4) are not exact solutions of the equations, but only balance certain terms that are asymptotically dominant. In the case of the pinch singularity these terms belong to surface tension, viscous, and inertial forces. Knowing that, dimensional analysis alone leads to the correct power laws. However, there are cases like the pinching of a very viscous thread (Papageorgiou (1995)), where the exponents are fixed to irrational values by other consistency requirements.

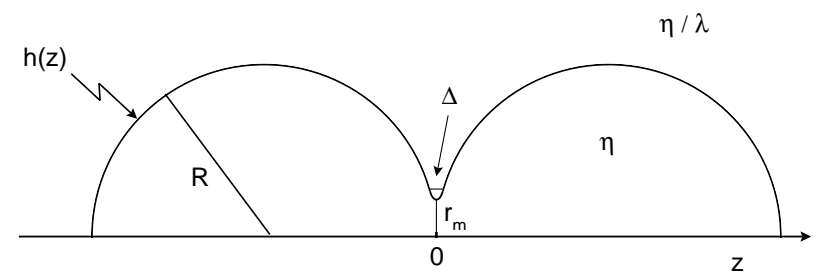

Fig. 2. Coalescence of two drops of radius $R$ and $R / \delta$. Asymptotically, the width $w$ of the bridge between the drops is much smaller than the minimum radius $r$.

What could possibly keep the local motion from behaving in a self-similar fashion? Two examples for broken self-similarity come from the coalescence of two drops. When two drops meet a point, surface tension will try to merge them into one drop, so the minimum radius $r$ of the bridge between the drops will increase, (Fig.2). As long as the bridge is very small, inertial effects can be neglected and the problem is initially governed by the Stokes equation. For geometrical reasons the width of the bridge $w$ is much smaller than the minimum radius $r$, so dimensionally

$$
r(t)=\frac{\gamma}{\eta} t
$$


is the only possible power law time dependence of the radius. However, a closer analysis reveals (Eggers, Lister, and Stone (1997)) that (5) has to be corrected by a $\operatorname{logarithmic}$ factor $\log (r / R)$, where $R$ is the initial radius of a drop. The reason for this change of the time dependence lies in the long-ranged character of the Stokes equation, which couples very disparate length scales. Hence the width of the bridge cannot be neglected, but enters as a logarithmic factor $\log (w / r) \sim$ $\log (r / R)$.

Another mechanism for broken self-similarity is observed for the same problem, but for much smaller initial size of the drops. In that case the surfacetension-driven motion will first occur on the surface alone, rather than being able to drive a flow in the interior. The equation of motion for this surface diffusion was first given by Mullins (1965). Based on simple scaling arguments, Mullins (1965) gave

$$
r(t) \sim t^{1 / 6}
$$

as the evolution of the radius, but this result could not be corroborated by his own numerical simulations. To understand this failure, one needs to take a closer look at the dynamics near the rising bridge (Eggers (1997b)). In contrast to the viscous flow problem, as the gap between the two spheres fills, a bulge of material forms just above the minimum. Eventually, it grows enough for both sides to touch, forming a void inside the material. Of course, at the point of touching a new singularity occurs and the topology of the problem has changed. The naive assumption of a single continuous evolution, underlying (6), is thus incorrect. Self-similarity can at best exist in a discrete sense.

\section{Birth of new structures}

If one drives the two-roller apparatus of Fig.1 much harder than necessary for the formation of a cusp, a second transition occurs, above which a thin sheet of air emanates from the cusp, and is drawn continuously into the fluid. This sheet is stable in time, but undergoes a three-dimensional instability at its lower end, where it decays into a curtain of tiny droplets. This provides a general mechanism for the entrainment of bubbles into a flow. The existence of the sheet was pointed out by Moffatt (1994), and confirmed in a series of qualitative experiments (Eggers and Smock (1996)), using a silicone oil 10000 times as viscous as water. At sufficiently high driving, the air forced into the cusp experiences a strong enough downward pull to form a stable sheet. A preliminary calculation suggests that the thickness of the sheet scales like

$$
\delta \approx\left(\frac{\eta_{\text {air }}}{\eta_{\text {fluid }}}\right)^{1 / 2} r_{c}
$$

This prediction is based on the assumption that there is a return flow in the sheet, which produces very high gradients. So when the sheet becomes very thin the inner flow is able to balance the high sheer stresses produced by the viscous flow. Both surface tension and gravity are not taken into account. 


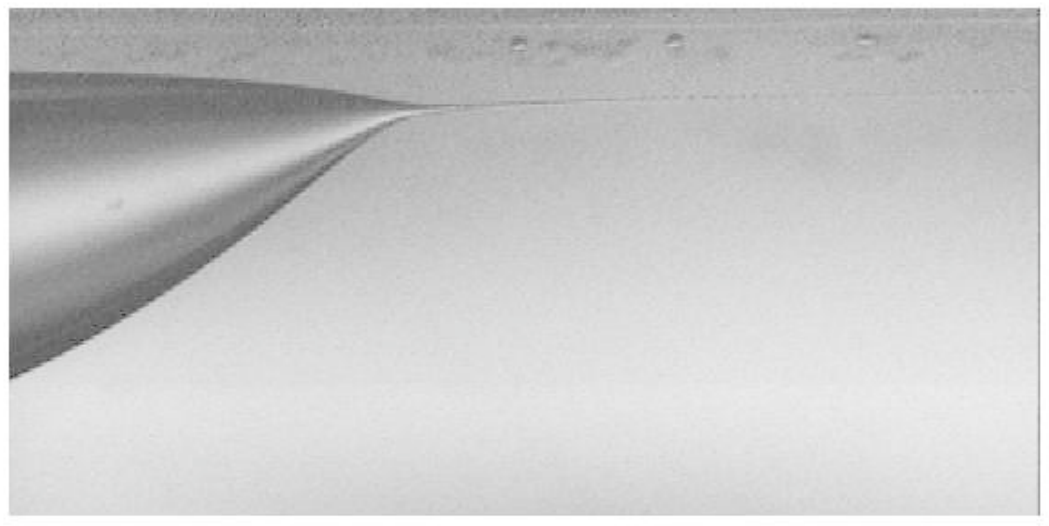

Fig. 3. Tip-streaming in a Couette device, showing a drop of water in silicone oil 1000 times as viscous. To initiate tip-streaming, $200 \mathrm{ppm}$ of surfactant has been added. The image height is $0.5 \mathrm{~mm}$. Photograph courtesy H. Leonhard.

Similar phenomena have been observed for a variety of other stationary singularities. An example, known as "tip-streaming" (Taylor (1934),De Bruijn (1993)), is shown in Fig.3. One sees a drop of water in a shear flow of a very viscous fluid, which produces a cone-shaped singularity at both ends of the drop. Under circumstances that are not well understood, a jet emanates from the tip, and eventually decays into drops due to the Rayleigh capillary instability. This is the precise analogue of the sheet in the two-roller apparatus, but the dimension of the singularity and of the resulting structure are lowered by one. A second example of a zero-dimensional singularity giving rise to a one-dimensional structure is that of a dielectric drop in a strong electric field, where a local cone-shaped solution exists (Taylor (1964)). This "Taylor cone" is never stable, but either oscillates between a rounded and a pointed state, or stabilizes itself by ejecting a jet from its tip. Again, little is known about the conditions under which the jet forms,but the striking similarities between different systems suggest a unifying explanation for the emergence of these structures.

\section{References}

Majda A. J. (1991): Vorticity, Turbulence, and Acoustics in Fluid Flow.

SIAM Review 33, 349-388

Dai W., Kadanoff L. P., Zhou S (1991): Interface dynamics and the motion of singularities. Phys. Rev. A 43, 6672-6682

Eggers J. (1997a): Nonlinear dynamics and breakup of free-surface flows.

Rev. Mod. Phys. 69, 865-929 
Brenner M. P., Shi X. D., Nagel S. R. (1994): Iterated Instabilities during Droplet Fission. Phys. Rev. Lett. 73, 3391-3394

Joseph D. D., Nelson J., Renardy M., Renardy Y. (1991): Two-dimensional cusped interfaces. J. Fluid Mech. 223, 383-409

Richardson S. (1968): Two dimensional bubbles in a slow flow.

J. Fluid Mech. 33, 475-493

Jeong J.-T., Moffatt H., K. (1992): Free-surface cusps associated with a flow at low Reynolds number. J. Fluid Mech. 241, 1-22

de Gennes P. G. (1985): Wetting: Statics and Dynamics. Rev. Mod. Phys. 57, 827-863

Brenner M., Bertozzi A. (1993): Spreading of droplets on a solid surface.

Phys. Rev. Lett. 71, 593-596

Papageorgiou D. T. (1995): On the breakup of viscous liquid threads.

Phys. Fluids 7, 1529-1544

Eggers E., Lister J., Stone H. (1997): Coalescence of liquid drops. manuscript in preparation

Mullins W. W., (1965): Morphological changes of a surface of revolution due to capillarity-induced surface diffusion. J. Appl. Phys. 36, 1826-1835

Eggers J., (1997b): Coalescence of spheres by surface diffusion.

Phys. Rev. Lett. 80, 2634-2637

Moffatt H. K. (1994): private communication

Eggers J., Smock M. (1996): The two-roller apparatus at very high driving. unpublished experiments

Taylor G. I. (1934): The formation of emulsions in definable fields of flow.

Proc. Roy. Soc. London A 146, 501-523

De Bruijn R. A.(1993): Tipstreaming of drops in simple shear flows.

Chem. Eng. Sci. 48, 277-284

Taylor G. I.(1964): Disentigration of water drops in an electric field.

Proc. Roy. Soc. London A 280, 383-397 